\title{
Structural covariance and cortical reorganization in schizophrenia: A MRI-based morphometric study
}

Lena Palaniyappan 1,2,3 Olha Hodgson ${ }^{4}$, Vijender Balain ${ }^{4}$ Sarina Iwabuchi ${ }^{4}$, Penny Gowland5 ${ }^{5}$ Peter Liddle 4 .

Running Title: Covariance and reorganization in schizophrenia

\section{Affiliations:}

${ }^{1}$ Robarts Research Institute \& The Brain and Mind Institute, University of Western Ontario, London, Ontario, Canada

2Department of Psychiatry, University of Western Ontario, London, Ontario, Canada 3Lawson Health Research Institute, London, Ontario, Canada.

4Translational Neuroimaging in Mental Health, University of Nottingham, UK 5Sir Peter Mansfield Imaging Center, University of Nottingham, Nottingham, UK

\section{Corresponding Author:}

Lena K. Palaniyappan

Prevention \& Early Intervention Program for Psychoses (PEPP) A2-636, LHSC-VH, 800 Commissioners Road, London, Ontario N6A 5W9. Canada Ph: (519) 685-8500 ext. 58054

Email: Ipalaniy@uwo.ca

Financial Support: Bucke Family Fund, Schulich School of Medicine, University of Western Ontario. Opportunities Fund, Academic Medical Organization of South Western Ontario. The data collection was supported by a Medical Research Council UK grant (G0601442). We acknowledge Dr. Mingli Li, Sichuan University for her assistance in preparing this manuscript. 
Conflicts of interest: LP received Travel Support from Magstim Limited (2014) and speaker fees from Otsuka (2017) and investigator-initiated grants from Otsuka Canada and Janssen Canada (2017). In the last 3 years, LP has held shares of Shire Inc. and Glaxo Smith Kline in his/his spousal pension funds. Other authors report no conflicts.

Word count (text body): 3763; Abstract word count: 232; Tables: 3; Figures:3; Supplemental Material: 1

\begin{abstract}
Background: In patients with schizophrenia, distributed abnormalities are observed in grey matter volume. A recent hypothesis posits that these distributed changes are indicative of a plastic reorganization process occurring in response to a functional defect in neuronal information transmission. We investigated the structural covariance across various brain regions in early-stage schizophrenia to determine if indeed the observed patterns of volumetric loss conform to a coordinated pattern of structural reorganization.
\end{abstract}

Methods: Structural MRI scans were obtained from 40 healthy adults and 41 age, gender and parental socioeconomic status matched patients with schizophrenia. Volumes of grey matter tissue was estimated at regional level across 90 atlas-based parcellations. Group level structural covariance was studied using a graph theoretical framework.

Results: Patients had distributed reduction in grey matter volume, with high degree of localized covariance (clustering) compared to controls. Patients with schizophrenia had reduced centrality of anterior cingulate and insula but increased centrality of the fusiform cortex, compared to controls. Simulating targeted removal of highly central nodes resulted in significant loss of the overall covariance patterns in patients compared to controls. 
Conclusion: Regional volumetric deficits in schizophrenia are not a result of random, mutually independent processes. Our observations support the occurrence of a spatially interconnected reorganization with systematic de-escalation of conventional 'hub' regions. This raises the question of whether the morphological architecture in schizophrenia is primed for compensatory functions, albeit with a high risk of inefficiency.

Key words: morphometry, cortical reorganization, grey matter, psychosis, schizophrenia

\section{Introduction}

Widespread reduction in grey matter volume notable even in the earliest stages of the illness (Tandon et al. 2008). Several meta-analyses of voxelwise morphometric studies in schizophrenia have concluded that the maximum likelihood of grey matter reduction is noted in the insula, anterior cingulate cortex, thalamus, superior temporal region and the hippocampal complex (Ellison-Wright et al. 2008; Glahn et al. 2008; Leung et al. 2009; Chan et al. 2011). Despite this, several studies report no notable structural alterations, especially when undertaking a whole-brain voxelwise search (mass univariate analysis), indicating that the regionally localized morphological abnormalities are subtle in magnitude (Fusar-Poli \& MeyerLindenberg 2016).

The application of graph theory to neuroimaging has enabled the study of entire brain as a network (the 'connectome') at a systems-level (Bullmore \& Sporns 2009). Specifically, when applied to morphometric data, graph-theory provides various metrics that quantify the degree of structural covariance among cortical regions 
within a group of subjects (Alexander-Bloch et al. 2013, p. ; Griffa et al. 2013). Measures of clustering and global efficiency reveal the localized (segregated) and distributed covariance patterns among brain regions. In addition to segregation and integration, measures of centrality in covariance networks reflect the relative importance of selected brain regions ('hubs') in 'influencing' or 'shaping' the observed relationships (Rubinov \& Sporns 2010). Furthermore, the dependence of a network on highly central regions ('hubs') can be inferred by studying the degree distribution (Achard et al. 2006). The robustness of a covariance network to withstand further loss of its component nodes (regions) can also be studied by simulating attacks on the nodes and estimating the effect of these attacks on the network parameters (Achard et al. 2006; van den Heuvel \& Sporns 2011). Earlier investigations of the structural covariance in schizophrenia have revealed a disturbance in regional relationships (increased segregation and reduced integration) (Bassett et al. 2008; Zhang et al. 2012).

Covariance among cortical regions reflects the 'common fate' of brain regions both in terms of coordinated maturation and combined plastic changes with training, experience or degeneration. In schizophrenia, while some structural changes occur even before the first episode, most occur in the first few years after the first episode, with a pattern of concomitant grey matter loss and relative increase compared to the baseline at the time of onset. We recently proposed that the extant findings of brain structural abnormalities in schizophrenia are suggestive of a post-onset cortical reorganization process wherein highly connected 'hub' regions are de-escalated, while peripheral hubs are super-delegated to compensate (Palaniyappan 2017). Such a pattern of topological decentralisation has been shown in structural (Crossley 
et al. 2014; Griffa et al. 2015) and functional connectivity networks (Lynall et al. 2010; Lo et al. 2015) in schizophrenia, but not studied to date in volumetric covariance networks. In the presence of grey matter reorganization, regional volumetric changes are unlikely to exhibit a random pattern; instead, we can expect a highly organized systematic change involving a redistribution of hubs.

In the present study, we used structural MRI data obtained from a sample of 81 subjects (41 patients, 40 controls) to study the properties of the schizophrenia connectome. We investigated if (1) volumetric changes in schizophrenia shows a systematic pattern of organized changes as opposed to random regional distribution (2) if there is a redistribution of the centrality (or primacy) of hub regions supporting the de-escalation hypothesis. We also studied the connectomic effect of further (simulated) tissue loss targeting prominent hub regions in patients compared to healthy controls.

\section{METHODS:}

\section{Subjects}

The data reported in the present study was obtained from a previously reported (Palaniyappan \& Liddle 2013) sample of 41 patients satisfying DSM-IV criteria for schizophrenia/schizoaffective disorder and 40 healthy controls. Patients were recruited from community-based mental health teams in Nottinghamshire and Leicestershire, United Kingdom. The diagnosis was made in a clinical consensus meeting in accordance with the procedure of Leckman et al. (1982), using all available information including a review of case files and a standardized clinical interview (Symptoms and Signs in Psychotic Illness (Liddle et al. 2002)). All patients 
were in a stable phase of illness with no change in antipsychotic-, antidepressant-, or mood-stabilizing medications in the 6 weeks prior to the study. Subjects with age $<18$ or $>50$, with neurological disorders, current substance dependence, or intelligence quotient $<70$ using Quick Test (Ammons \& Ammons 1962) were excluded. . The median Defined Daily Dose (DDD)(WHO Collaborating Centre for Drug Statistics and Methodology 2003) was calculated for all prescribed psychotropic medications.

Healthy controls were recruited from the local community via advertisements, and 40 subjects free of any psychiatric or neurological disorder group-matched for age and parental socioeconomic status (measured using National Statistics - Socio Economic Classification (Rose \& Pevalin 2003)) included to the patient group. Controls had similar exclusion criteria to patients; in addition, subjects with personal or family history of psychotic illness were excluded. A clinical interview by a research psychiatrist was employed to ensure that the controls were free from current axis 1 disorder and history of either psychotic illness or neurological disorder. The study was given ethical approval by the National Research Ethics Committee, Derbyshire, United Kingdom. All volunteers gave written informed consent. Please see Table 1 for further sample characteristics.

Table 1 here

\section{Image acquisition and processing}

A magnetization-prepared rapid acquisition gradient echo image with $1 \mathrm{~mm}$ isotropic resolution, $256 \times 256 \times 160$ matrix, Repetition Time (TR)/Echo Time (TE) $8.1 / 3.7 \mathrm{~ms}$, shot interval $3 \mathrm{~s}$, flip angle $8^{\circ}$, SENSE factor 2 was also acquired for each participant 
for reconstructing the anatomical surface. T1 images were resliced (1 $\mathrm{mm}$ isotropic) and segmented into grey, white and CSF tissue using the SPM8 Diffeomorphic Anatomical Registration Through Exponentiated Lie algebra (DARTEL) algorithm (Ashburner 2007). GM images were normalized to MNI space. The normalized, modulated, unsmoothed GM images were then used as inputs for the construction of graph networks.

\section{Constructing covariance networks}

All topological properties were computed using Graph Analysis Toolbox(GAT) (Hosseini et al. 2012) (http://brainlens.org/tools.html) that uses computation algorithms from Brain Connectivity Toolbox (https://sites.google.com/site/bctnet/). In line with previous works ((Achard et al. 2006; Singh et al. 2013) we generated 90 cortical and subcortical regions using the AAL-90 atlas. Using linear regression model with age, gender and intracranial volume as covariates, residuals of regional volumes were extracted. We chose these covariates for adjustment, as prior studies have demonstrated their confounding potential (Mechelli et al. 2005; Modinos et al. 2009; Montembeault et al. 2012; Li et al. 2013). A 90X90 Pearson's correlation matrix of residuals of grey matter volumes of the 90 parcellated brain regions was used to create a binary adjacency matrix for each group (CON and SCZ). We used a range of thresholds determined by connection densities (proportions of connections present in a graph to all possible connections) varying from 0.3 to 0.5 (increments of $0.025)$ to compare the properties of emerging networks. Across this range in both groups, the resulting graphs were fully connected and not fragmented (minimum density at which fully connected graph was observed $=0.29$ ). The graphs lose sparsity and develop a random configuration when $>50 \%$ of all possible edges are 
retained (a cost density of 0.5 ). The steps involved in obtaining the connectomes are summarized in Figure 1. The connectomes were visualized using BrainNet Viewer (Xia et al. 2013) (http://www.nitrc.org/projects/bnv/).

\section{Integration, segregation and centrality}

The patterns of relationship among brain regions within in a network can be described using three groups of topological properties (integration, segregation and centrality) that can be quantified using various graph theoretical measures (Stam \& Reijneveld 2007; Bullmore \& Sporns 2009; Rubinov \& Sporns 2010), as described below.

(1) Integration: Shortest path length $L_{p}$ between two regions $(A, B)$ refers to the minimum number of node-to-node edges that connect $A$ and $B$. The average shortest path length between all pairs of regions in the network gives the characteristic path length of the network $\left(M L_{p}\right)$. The inverse of $M L_{p}$ is a measure of

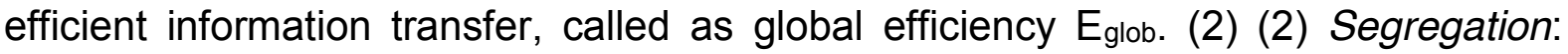
Clustering coefficient $C_{p}$ indicates the presence of high degree of covariance (number of connections or edges) among neighbouring regions. The average of clustering coefficients of each region (or node) provides the clustering coefficient of the network $\left(M C_{p}\right)$. Local efficiency of a region, $E_{l o c}$, is a closely related metric given by the inverse of the minimum number of connections among each pair of neighbouring regions. $C_{p}$ and $E_{\text {loc }}$ quantify the cliquishness of a region.

(3) Centrality: The degree (number of connections) of a region (or node) is a sensitive measure of centrality for structural networks (Rubinov \& Sporns 2010). In line with previous connectomic studies (Palaniyappan et al. 2014, 2016), we estimated the small-world index by comparing the estimated topological properties 
$\left(\mathrm{MC}_{\mathrm{p} \text { and }} \mathrm{ML} \mathrm{L}_{\mathrm{p}}\right)$ of the two networks (CON and SCZ) with corresponding mean values of 20 null random graphs ( $\mathrm{MC}_{\text {null }}$ and $\mathrm{MLnull}$ ) constructed with same number of nodes, edges and degree distribution as the volume based networks. Small world index (SWI) is given by ([MCp/ MC null]/[ MLp/ MLnull]. SWI>1 suggests a small world network that has a relatively high segregation and integration compared to random null networks (Humphries \& Gurney 2008). Further, we also used Newman's optimization algorithm (Newman 2006) implemented in GAT with 1000 iterations to identify the modular organization in the CON and SCZ connectomes. Modules are defined as a subgroup of regions that have higher covariance within the subgroup, than their covariance with regions outside the subgroup.

\section{Resilience}

Small-world structural brain networks follow an exponentially truncated power-law function for cumulative degree distribution that can be expressed as $P(d) \sim\left[d^{(k-1) *} e^{(-}\right.$ $\left.{ }^{d / d c}\right)$, where $P(d)$ is the probability of regional degree $(d)$, dc is the cut-off degree above which there is an exponential decay in probability of hubs (i.e. regions with degree $>2$ s.d. units), ' $k$ ' being the estimated component. If such a truncated powerlaw relationship can be demonstrated for a connectome, this will indicate a scaling regimen that permits the presence of high degree hubs but constrains against the emergence of 'mega-hubs' that are connected to a very large number of regions. Such a constrained network is more resilient to the removal of both high degree hubs and random hubs when compared to scale-free networks (such as the world-wide web (Barabasi 2009)). We initially tested if this assumption is true for both groups.

Following this, we assessed the resilience of the connectome using the approach adopted by Achard et al. (2006). Random attack involved the removal of one random 
regional node (and its connections) from a network and calculating the size of the largest connected component (i.e. a fully connected graph from the remaining nodes) and global efficiency of the network. Each random removal was done 100 times and the average measures of the remaining graph were computed. This process was repeated until a path length of 1 was reached. In targeted attack, nodes were removed in the order of their relative degree i.e. the first attack was on the most central hub in the network, subsequent removals progressed in a descending order of normalized nodal degree. The attacks were carried out separately for the networks obtained from each group at the minimum density for full connectivity and the resulting plots were compared as described below.

\section{Group comparison}

To test the statistical significance of the difference between the topological parameters of the two groups, non- parametric permutation testing with 1000 repetitions was employed. For each iteration, the corrected grey matter volumes of each participant were randomly reassigned to one of two new groups with the sample size identical as controls and patients. Binary adjacency matrices across a range of network densities ( 0.3 to 0.5 , increments of 0.025$)$ were obtained for each randomized group. Topological measures were then calculated for the networks and differences between the random groups were computed across the entire range of densities. For the various topological properties, differences in the area under the curves obtained from plotting the values of each random group across the range of densities was obtained for each iteration. This resulted in a null distribution of differences, against which the $p$ values of the actual differences in the curve 
functions obtained by comparing $\mathrm{CON}$ and $\mathrm{SCZ}$ were computed. This nonparametric permutation test based on functional data analysis (FDA) (Ramsay \& Dalzell 1991) that compares the functions of the curve obtained across thresholds in one group with the curve from the other group. As opposed to multiple tests comparing means at each threshold, the use of a single test comparing curve functions (FDA), requires no further multiple test correction for the number of threshold points that are studied. For regional ( $\mathrm{n}=90$ nodes) properties such as local efficiency, clustering and degree, an additional correction for multiple comparison (false discovery rate) was used with corrected two-tailed $p<0.05$ considered as significance threshold. This multiple testing correction (FDR) is done across the number of nodes (90). The same permutation approach was also used when comparing the curves obtained from random and targeted attack on $\mathrm{CON}$ and $\mathrm{SCZ}$ networks. Hubs were defined as the nodes whose FDA-based curve function for regional degree is 1 standard deviation (Bassett et al. 2008; Hosseini et al. 2013) greater than the mean of corresponding curve functions obtained from the 1000 random permutations.

\section{Results}

\section{Global properties}

Both control and schizophrenia connectomes showed small-worldness (mean SWI across densities for $\mathrm{CON}=1.012$; SCZ=1.068). Patients had significantly higher clustering and a trend towards lower global efficiency (Table 2). For both controls and patients, we observed an exponentially truncated power-law distribution. The exponent estimate (k) was 1.05 in patients and 1.28 in controls. The cut-off degree (dc) was 9.02 for patients and 5.93 for controls. The R-square value for the 
distribution fit was 0.88 for patients and 0.86 for controls, suggesting that the truncated power-law model had very good fit for the data as expected.

\section{Resilience}

There was a significant reduction in the resilience of the SCZ connectome to targeted attack, but not random attack, when compared to controls. When compared to controls, random attack produced a $1.8 \%$ reduction in the size of the largest connected component and $1.24 \%$ reduction in global efficiency; but targeted removal of hubs produced a $14.2 \%$ ( $>7$ times more) reduction in the largest connected component and $13.23 \%$ (>10 times more) reduction in global efficiency in patients. The degree distribution plots and the results of simulation analysis are shown in Figure 2.

Figure 2 here

Table 2 here

\section{Regional Integration, segregation and centrality}

Examination of the individual nodal properties revealed significantly reduced clustering coefficient in right middle temporal region $(p=0.028)$ and reduced local efficiency in right hippocampus $(p=0.034)$ and right anterior cingulate cortex $(p=0.046)$ in patients compared to controls. Nodal degree was significantly reduced for right insula $(p=0.038)$ and left middle (dorsolateral) frontal cortex $(p=0.002)$ in patients. These results are summarized in figure 3 . In both patients and controls, hub regions were predominantly located in the frontal cortex and were mostly comparable in the two groups (Table 3). Notably, among frontal hubs, anterior 
cingulate and gyrus rectus showed high degree in controls, though did not emerge as hubs in patients. Among the non-frontal hubs, insula was a prominent hub in controls but not in patients, while fusiform region was a hub in patients but not in controls.

Figure 3 here

Tables 3 here

\section{Module membership}

The distribution of the module membership in controls revealed 5 prominent modules (a large fronto-insular, a temporal, an occipital, a parietal and a subcortical module). In patients, the optimal solution yielded 7 modules. Lobar partitioning was less clearcut in patients when compared to controls. Most notably, the subcortical module was split with bilateral thalami being separated from the rest of the modules. Similarly, bilateral superior parietal regions appeared as a separate module. The modular structure of the connectome is shown in supplemental figure and table S1. We also present the VBM findings (controls vs. patients) in the supplement.

\section{Discussion}

Using a connectomic approach on morphometric data, we observe that the structural covariance of grey matter volume in patients with schizophrenia is not random, but 
significantly deviates from healthy controls. An increase in overall clustering (i.e. constrained covariance) despite reduced clustering of certain brain regions (anterior cingulate, middle temporal cortex and hippocampus), reduced centrality of the insula and dorsolateral frontal cortex along with a modular segregation of thalamus and superior parietal regions was seen in schizophrenia. The overall increase in segregation and the trend towards reduced global efficiency is consistent with several other connectomic studies in schizophrenia (van den Heuvel et al. 2010; Fornito et al. 2012; Griffa et al. 2013)(Fornito et al. 2012)(Bassett et al. 2008; Alexander-Bloch et al. 2010; Lynall et al. 2010; Zhang et al. 2012) (Wang et al. 2012).

All of the regional nodes showing altered topological properties in this study are implicated in the structural alterations seen in schizophrenia (Ellison-Wright et al. 2008; Glahn et al. 2008; Palaniyappan \& Liddle 2012). While we observed grey matter reduction in many of these regions (thalamus, insula, superior temporal gyrus, hippocampus; See Table S3) in a VBM analysis at a lenient threshold suitable for defining discrete clusters and investigate specific regions of interest, these VBM differences did not survive conventional correction for multiple testing (FDR<0.05). The observed reduction in regional topological properties (degree of dorsolateral prefrontal cortex and insula and local efficiency of anterior cingulate cortex and hippocampus) despite having only weakly localisable regional structural changes indicates that the graph-based measures are of larger magnitude of effect. These findings are consistent with Chen et al.'s (2014) who reported limited VBM-based regional GM deficits, but a pronounced deviation of structural covariance in schizophrenia. 
Our observations replicate previous findings that the structural covariance in schizophrenia exhibits small-world properties (Bassett et al. 2008; Zhang et al. 2012). In addition, for the first time, we show that the degree distribution of the structural connectome in patients follows an exponentially truncated power-law function. This pattern of degree distribution suggests that despite the volumetric deficits that occur, certain brain regions emerge with very high degree (i.e. supercovarying mega-hubs). In the context of reduced centrality of traditional 'hub' regions (i.e. dorsolateral prefrontal, insula, anterior cingulate), the emergence of peripheral mega-hubs (e.g. fusiform) supports the possibility of inefficient cortical reorganization that is unlikely to be advantageous if further plastic changes affect the function of these central nodes (Bullmore et al. 2009).

Exponentially truncated degree distribution has been previously noted in the functional connectome in a sample of 12 patients with schizophrenia (Lynall et al. 2010), but in contrast to Lynall et al. who observed a lower cut-off of the degree at which exponential decay in the probability of hubs in patients, we noted somewhat higher cut-off degree in patients. This difference could be attributed to the differing properties of the resting-state functional connectome when compared to a morphological covariance network. Our observation suggests that the structural network in schizophrenia shows a subtle shift towards a scale-free organization. There are important implications of this observation for a disease state such as schizophrenia that involves grey matter reduction. Most brain regions in a scale-free network have very few covarying links, so if the disease process affects nodes in a non-selective fashion (random or generalized reduction in volume), the network can 
still function efficiently (Bullmore et al. 2009). However, upon focused removal of high degree nodes, a scale-free network will become inefficient rapidly.

For the first time, using simulated targeted and random attacks, we demonstrate this over-reliance on hubs affecting the volumetric covariance in schizophrenia. One major implication of this finding is that continued tissue loss affecting hub regions could compromise the structural covariance, affecting the putative structural reorganization process. Notably, we reported functional topological decentralization and the emergence of peripheral hubs using task-based and resting state fMRI in this sample of patients previously (Palaniyappan \& Liddle 2013), supporting the notion that functional connectivity changes may underlie structural covariance. Extrapolating this observation, we speculate the overreliance on non-conventional 'mega' hubs of structural covariance represent the effect of functional reorganization, whereby rapid functional synchronization or percolation of information across the entire network is facilitated in the wake of synaptic inefficiency producing a bottleneck effect at traditional hub regions (Palaniyappan 2017). While this may be advantageous (and possibly aid in recovery) in some cases, reflecting a compensation process, by altering regional functional specialization, the putative reorganization may prompt inappropriate information transfer, and inefficient recruitment of extant brain regions when cognitive demands arise. Studying the longitudinal relationship between functional dysconnectivity and grey matter reorganization is required to refute or prove the efficiency and compensatory effect of the presumed reorganization. 
Several limitations must be borne in mind while interpreting these results. Firstly, as our approach of estimating structural covariance is based on between-subjects variance, we are not able to directly relate the reported topological metrics to individual differences in individual measurements of brain function. This limited our inferences on the direct functional implications of our findings. We recruited a medicated sample of patients with schizophrenia; grey matter changes are noted to be more prominent in patients with established illness who are taking antipsychotic medications (Leung et al. 2009; Ho et al. 2011). While it is not possible to separate the effect of antipsychotic induced changes from those that result from an inherent disease process, at least the linear effect of current antipsychotic dose on the topological properties of structural connectome appears to be negligible (supplemental material). Ideally, longitudinal data on initially unmedicated sample is required to investigate this issue. There are several uncertain variables when constructing graph-based networks. This includes the dependency of several topological properties on the size of the selected nodes and the threshold used for binarisation (Fornito et al. 2013; Zalesky et al. n.d.). While the results of group comparisons on the basis of uniform node selection and thresholding process has been shown to yield broadly consistent results (Evans 2013), absolute values of the network parameters must be cautiously interpreted. Finally, we interpret the results based on the theoretical framework of neuroplasticity; other explanations (e.g. neuroprogression or neurodevelopmental defect) cannot be ruled out from the presented data. It is worth noting that in the same sample, gyrification based covariance showed no overall differences in segregating or integration, though regional topological changes were present (Palaniyappan et al. 2014). 
Structural covariance reported here suggests that a system-level disturbance in morphology that is possibly related to coordinated maturation or plasticity of brain regions can be observed despite subtle regionally localised structural changes in schizophrenia. This raises the possibility that tissue preservation aimed at specific regions might have a wider impact on reversing or preventing the observed abnormalities. Importantly, this study highlights the importance of the crucial hub regions in influencing the overall topological architecture observed in patients. It is tempting to speculate that tissue preservation (or plasticity modulation) strategies that focus on these central nodes could favorably alter the cortical reorganization process in schizophrenia. Several encouraging tissue preservation strategies have been previously suggested, with specific regional effects. Further environmental risk factors such as cannabis affect key hub regions such as the hippocampus (Rapp et al. 2012). In addition to reducing cannabis, physical exercise (Pajonk et al. 2010) and cognitive-enhancement therapy (Eack et al. 2010) can help to preserve the grey matter volume of hippocampus; specific cognitive training such as mindfulness mediation could affect insular structure (Luders et al. 2012); attentional training (Hoekzema et al. 2011) could have a positive impact on frontal volume; neuromodulation techniques such as transcranial magnetic stimulation could have a positive impact on temporal volume (May et al. 2007). Future studies investigating the impact of these strategies on the structural connectome could support or refute our optimistic conclusions.

\section{REFERENCE}

Achard S, Salvador R, Whitcher B, Suckling J, Bullmore E (2006). A resilient, low-frequency, small-world human brain functional network with highly connected association cortical hubs. The Journal of neuroscience: the official journal of the Society for Neuroscience 26, 63-72. 
Alexander-Bloch A, Giedd JN, Bullmore E (2013). Imaging structural co-variance between human brain regions. Nature reviews. Neuroscience 14, 322-336.

Alexander-Bloch AF, Gogtay N, Meunier D, Birn R, Clasen L, Lalonde F, Lenroot R, Giedd J, Bullmore ET (2010). Disrupted Modularity and Local Connectivity of Brain Functional Networks in Childhood-Onset Schizophrenia. Frontiers in Systems Neuroscience 4

Ammons RB, Ammons CH (1962). The Quick Test (QT): Provisional manual. Psychological Reports 11, 111-161.

Ashburner J (2007). A fast diffeomorphic image registration algorithm. NeuroImage 38, 95-113.

Barabasi A-L (2009). Scale-Free Networks: A Decade and Beyond. Science 325, 412-413.

Bassett DS, Bullmore E, Verchinski BA, Mattay VS, Weinberger DR, Meyer-Lindenberg A (2008). Hierarchical Organization of Human Cortical Networks in Health and Schizophrenia. J. Neurosci. 28, 92399248.

Bullmore E, Barnes A, Bassett DS, Fornito A, Kitzbichler M, Meunier D, Suckling J (2009). Generic aspects of complexity in brain imaging data and other biological systems. NeuroImage 47, 1125-1134.

Bullmore E, Sporns O (2009). Complex brain networks: graph theoretical analysis of structural and functional systems. Nat Rev Neurosci 10, 186-198.

Chan RCK, Di X, McAlonan GM, Gong Q (2011). Brain anatomical abnormalities in high-risk individuals, first-episode, and chronic schizophrenia: an activation likelihood estimation meta-analysis of illness progression. Schizophrenia bulletin 37, 177-188.

Chen Z, Deng W, Gong Q, Huang $C$, Jiang $L$, Li M, He Z, Wang $Q$, Ma $X$, Wang $Y$, Chua SE, McAlonan GM, Sham PC, Collier DA, McGuire $P$, Li T (2014). Extensive brain structural network abnormality in firstepisode treatment-naive patients with schizophrenia: morphometrical and covariation study. Psychological Medicine 44, 2489-2501.

Crossley NA, Mechelli A, Scott J, Carletti F, Fox PT, McGuire P, Bullmore ET (2014). The hubs of the human connectome are generally implicated in the anatomy of brain disorders. Brain: A Journal of Neurology 137, 2382-2395.

Eack SM, Hogarty GE, Cho RY, Prasad KMR, Greenwald DP, Hogarty SS, Keshavan MS (2010). Neuroprotective effects of cognitive 
enhancement therapy against gray matter loss in early schizophrenia: results from a 2-year randomized controlled trial. Archives of General Psychiatry 67, 674-682.

Ellison-Wright I, Glahn DC, Laird AR, Thelen SM, Bullmore E (2008). The Anatomy of First-Episode and Chronic Schizophrenia: An Anatomical Likelihood Estimation Meta-Analysis. Am J Psychiatry 165, 1015-1023.

Evans AC (2013). Networks of anatomical covariance. NeuroImage 80, 489-504.

Fornito A, Zalesky A, Breakspear M (2013). Graph analysis of the human connectome: Promise, progress, and pitfalls. NeuroImage $\mathbf{8 0 C}$, 426-444.

Fornito A, Zalesky A, Pantelis C, Bullmore ET (2012). Schizophrenia, neuroimaging and connectomics. NeuroImage

Fusar-Poli P, Meyer-Lindenberg A (2016). Forty years of structural imaging in psychosis: promises and truth. Acta Psychiatrica Scandinavica 134, 207-224.

Glahn DC, Laird AR, Ellison-Wright I, Thelen SM, Robinson JL, Lancaster JL, Bullmore E, Fox PT (2008). Meta-Analysis of Gray Matter Anomalies in Schizophrenia: Application of Anatomic Likelihood Estimation and Network Analysis. Biological Psychiatry 64, 774-781.

Griffa A, Baumann PS, Ferrari C, Do KQ, Conus P, Thiran J-P, Hagmann P (2015). Characterizing the connectome in schizophrenia with diffusion spectrum imaging. Human Brain Mapping 36, 354-366.

Griffa A, Baumann PS, Thiran J-P, Hagmann P (2013). Structural connectomics in brain diseases. NeuroImage 80,515-526.

van den Heuvel MP, Mandl RCW, Stam CJ, Kahn RS, Hulshoff Pol HE (2010). Aberrant frontal and temporal complex network structure in schizophrenia: a graph theoretical analysis. The Journal of neuroscience: the official journal of the Society for Neuroscience 30, 15915-15926.

van den Heuvel MP, Sporns 0 (2011). Rich-club organization of the human connectome. The Journal of neuroscience: the official journal of the Society for Neuroscience 31, 15775-15786.

Ho B-C, Andreasen NC, Ziebell S, Pierson R, Magnotta V (2011). Long-term antipsychotic treatment and brain volumes: a longitudinal study of first-episode schizophrenia. Archives of General Psychiatry 68, 128-137. 
Hoekzema E, Carmona S, Ramos-Quiroga JA, Barba E, Bielsa A, Tremols V, Rovira M, Soliva JC, Casas M, Bulbena A, Tobeña A, Vilarroya $\mathbf{O}$ (2011). Training-induced neuroanatomical plasticity in ADHD: a tensor-based morphometric study. Human brain mapping 32, 1741-1749.

Hosseini SMH, Black JM, Soriano T, Bugescu N, Martinez R, Raman MM, Kesler SR, Hoeft F (2013). Topological properties of large-scale structural brain networks in children with familial risk for reading difficulties. NeuroImage 71, 260-274.

Hosseini SMH, Hoeft F, Kesler SR (2012). GAT: a graph-theoretical analysis toolbox for analyzing between-group differences in large-scale structural and functional brain networks. PloS one 7, e40709.

Humphries MD, Gurney K (2008). Network "small-world-ness": a quantitative method for determining canonical network equivalence. PloS one 3, e0002051.

Leckman JF, Sholomskas D, Thompson D, Belanger A, Weissman MM (1982). Best Estimate of Lifetime Psychiatric Diagnosis: A Methodological Study. Arch Gen Psychiatry 39, 879-883.

Leung M, Cheung C, Yu K, Yip B, Sham P, Li Q, Chua S, McAlonan G (2009). Gray Matter in First-Episode Schizophrenia Before and After Antipsychotic Drug Treatment. Anatomical Likelihood Estimation Metaanalyses With Sample Size Weighting. Schizophr Bull, sbp099.

Li X, Pu F, Fan Y, Niu H, Li S, Li D (2013). Age-related changes in brain structural covariance networks. Frontiers in Human Neuroscience $\mathbf{7}$

Liddle PF, Ngan ETC, Duffield G, Kho K, Warren AJ (2002). Signs and Symptoms of Psychotic Illness (SSPI): a rating scale. The British Journal of Psychiatry 180, 45-50.

Lo C-YZ, Su T-W, Huang C-C, Hung C-C, Chen W-L, Lan T-H, Lin C-P, Bullmore ET (2015). Randomization and resilience of brain functional networks as systems-level endophenotypes of schizophrenia. Proceedings of the National Academy of Sciences of the United States of America 112, 9123-9128.

Luders E, Mayer EA, Toga AW, Narr KL, Gaser C (2012). The unique brain anatomy of meditation practitioners: alterations in cortical gyrification. Frontiers in Human Neuroscience 6, 34.

Lynall M-E, Bassett DS, Kerwin R, McKenna PJ, Kitzbichler M, Muller U, Bullmore E (2010). Functional Connectivity and Brain Networks in Schizophrenia. J. Neurosci. 30, 9477-9487. 
May A, Hajak G, Gänßbauer S, Steffens T, Langguth B, Kleinjung T, Eichhammer P (2007). Structural Brain Alterations following 5 Days of Intervention: Dynamic Aspects of Neuroplasticity. Cerebral Cortex 17, 205-210.

Mechelli A, Friston KJ, Frackowiak RS, Price CJ (2005). Structural Covariance in the Human Cortex. Journal of Neuroscience 25, 8303-8310.

Modinos G, Vercammen A, Mechelli A, Knegtering H, McGuire PK, Aleman A (2009). Structural covariance in the hallucinating brain: a voxel-based morphometry study. 34, 465-469.

Montembeault M, Joubert S, Doyon J, Carrier J, Gagnon J-F, Monchi O, Lungu O, Belleville S, Brambati SM (2012). The impact of aging on gray matter structural covariance networks. NeuroImage 63, 754-759.

Newman MEJ (2006). Finding community structure in networks using the eigenvectors of matrices. Physical review. E, Statistical, nonlinear, and soft matter physics 74, 36104.

Pajonk F-G, Wobrock T, Gruber O, Scherk H, Berner D, Kaizl I, Kierer A, Muller S, Oest M, Meyer T, Backens M, Schneider-Axmann T, Thornton AE, Honer WG, Falkai P (2010). Hippocampal Plasticity in Response to Exercise in Schizophrenia. Arch Gen Psychiatry 67, 133-143.

Palaniyappan L (2017). Progressive cortical reorganisation: A framework for investigating structural changes in schizophrenia. Neuroscience \& Biobehavioral Reviews

Palaniyappan L, Liddle PF (2012). Differential effects of surface area, gyrification and cortical thickness on voxel based morphometric deficits in schizophrenia. NeuroImage 60, 693-699.

Palaniyappan L, Liddle PF (2013). Diagnostic Discontinuity in Psychosis: A Combined Study of Cortical Gyrification and Functional Connectivity. Schizophrenia bulletin

Palaniyappan L, Marques TR, Taylor $H$, Mondelli V, Reinders AATS, Bonaccorso S, Giordano A, DiForti M, Simmons A, David AS, Pariante CM, Murray RM, Dazzan P (2016). Globally Efficient Brain Organization and Treatment Response in Psychosis: A Connectomic Study of Gyrification. Schizophrenia Bulletin

Palaniyappan L, Park B, Balain V, Dangi R, Liddle P (2014). Abnormalities in structural covariance of cortical gyrification in schizophrenia. Brain structure \& function 
Ramsay JO, Dalzell CJ (1991). Some tools for functional data analysis. Journal of the Royal Statistical Society. Series B. Methodological 53, 539572.

Rapp C, Bugra H, Riecher-Rossler A, Tamagni C, Borgwardt S (2012). Effects of Cannabis Use on Human Brain Structure in Psychosis: A Systematic Review Combining In Vivo Structural Neuroimaging and Post Mortem Studies. Current Pharmaceutical Design 18, 5070-5080.

Rose D, Pevalin DJ (2003). A Researcher's Guide to the National Statistics Socio-economic Classification. Sage Publications: London.

Rubinov M, Sporns O (2010). Complex network measures of brain connectivity: Uses and interpretations. NeuroImage 52, 1059-1069.

Singh MK, Kesler SR, Hadi Hosseini SM, Kelley RG, Amatya D, Hamilton JP, Chen MC, Gotlib IH (2013). Anomalous Gray Matter Structural Networks in Major Depressive Disorder. Biological psychiatry

Stam C, Reijneveld J (2007). Graph theoretical analysis of complex networks in the brain. Nonlinear Biomedical Physics 1, 3.

Tandon R, Keshavan MS, Nasrallah HA (2008). Schizophrenia, 'Just the Facts': what we know in 2008 part 1: overview. Schizophrenia Research 100, 4-19.

Wang Q, Su T-P, Zhou Y, Chou K-H, Chen I-Y, Jiang T, Lin C-P (2012). Anatomical insights into disrupted small-world networks in schizophrenia. NeuroImage 59, 1085-1093.

WHO Collaborating Centre for Drug Statistics and Methodology (2003). Guidelines for ATC Classification and DDD Assignment. . WHO Collaborating Centre for Drug Statistics and Methodology

Xia M, Wang J, He Y (2013). BrainNet Viewer: A Network Visualization Tool for Human Brain Connectomics. PloS one 8, e68910.

Zalesky A, Fornito A, Harding IH, Cocchi L, Yucel M, Pantelis C, Bullmore ET (n.d.). Whole-BrainAnatomicalNetworks: Does the choice of nodesmatter? NeuroImage In Press, Accepted Manuscript

Zhang $Y$, Lin L, Lin C-P, Zhou Y, Chou K-H, Lo C-Y, Su T-P, Jiang T (2012). Abnormal topological organization of structural brain networks in schizophrenia. Schizophrenia research 141, 109-118. 
Figure Captions:

Figure 1: The steps involved in generating group-based volumetric connectomes A.T1 MPRAGE images resliced and segmented into probabilistic grey, white maps and cerebrospinal fluid using SPM8 software. The grey matter maps were parcellated into 90 cortical and subcortical regions using the AAL atlas. B. Groupwise $90 \times 90$ correlation matrices created by calculating the correlations between the probabilistic grey matter volume in each parcellated brain region. C. Binary adjacency matrices were derived from thresholding at a range of densities (min 0.3 , max 0.5 , interval 0.025 ) for fully connected graphs in both groups. D. Topological properties of the connectome were computed using Graph Analysis Toolbox - GAT (Hosseini et al., 2012) and visualized using BrainNet Viewer (Xia et al., 2013)

Figure 2: Results of simulated removal of nodes from covariance networks in patients and controls. Results of random [left column] and targeted [right] attack are shown. In the top panel, the size of the largest connect component (LCC), is plotted against the fraction of removed nodes. In the middle panel, global efficiency (Geff), is plotted against the fraction of removed nodes. The bottom panel displays the degree distribution of nodes in both groups.

Figure 3: Regional changes in the network properties of the gray matter connectome in schizophrenia compared to controls. Nodal degree was significantly reduced for left middle (dorsolateral) frontal cortex $(A)(p=0.002)$ and right insula $(B)(p=0.038)$; Clustering coefficient reduced in right middle temporal region $(C)(p=0.028)$ and local 
efficiency reduced in right anterior cingulate cortex (D) $(p=0.034)$ and right hippocampus $(E)(p=0.046)$ in patients compared to controls. 


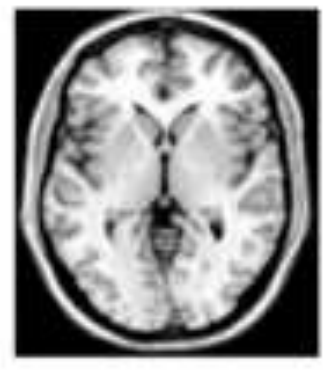

\section{A. Atlas-based image parcellation}
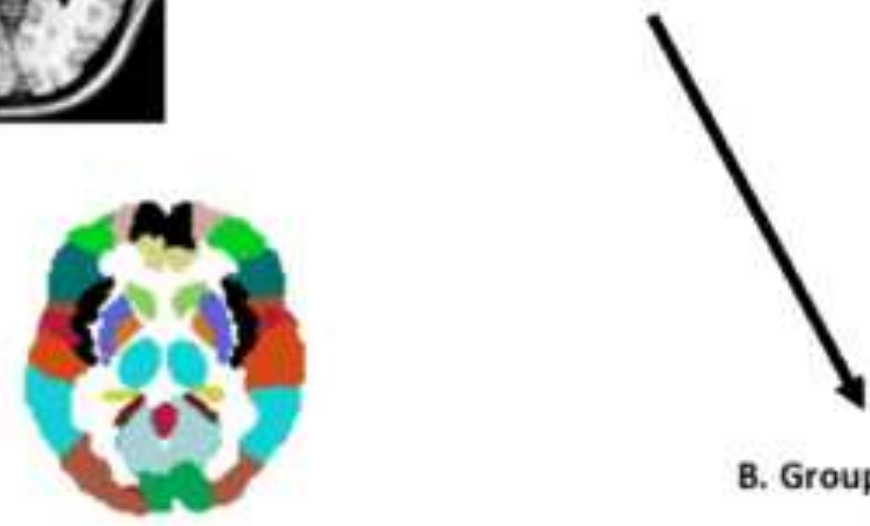

B. Group-wise $90 \times 90$ correlation matrices
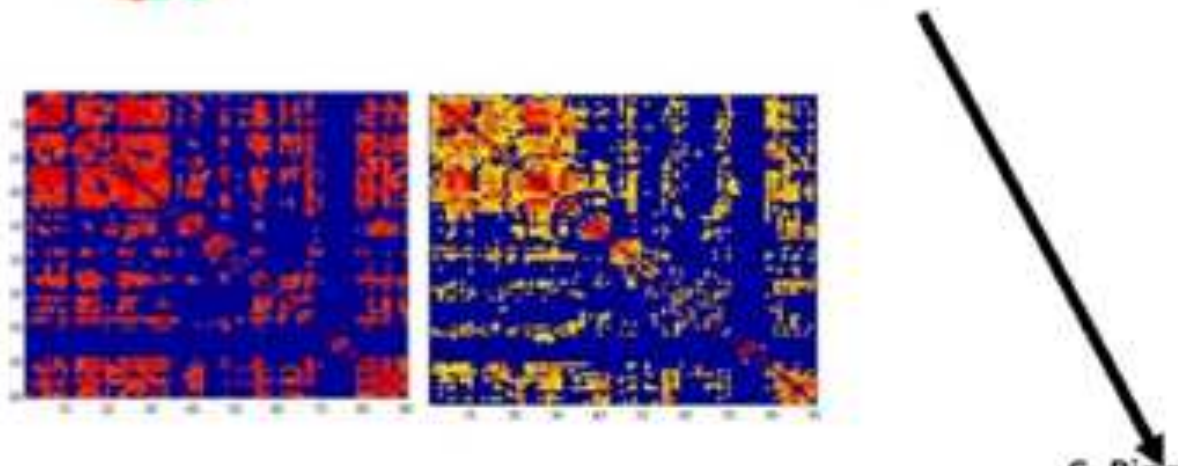

C. Binarisation across densities
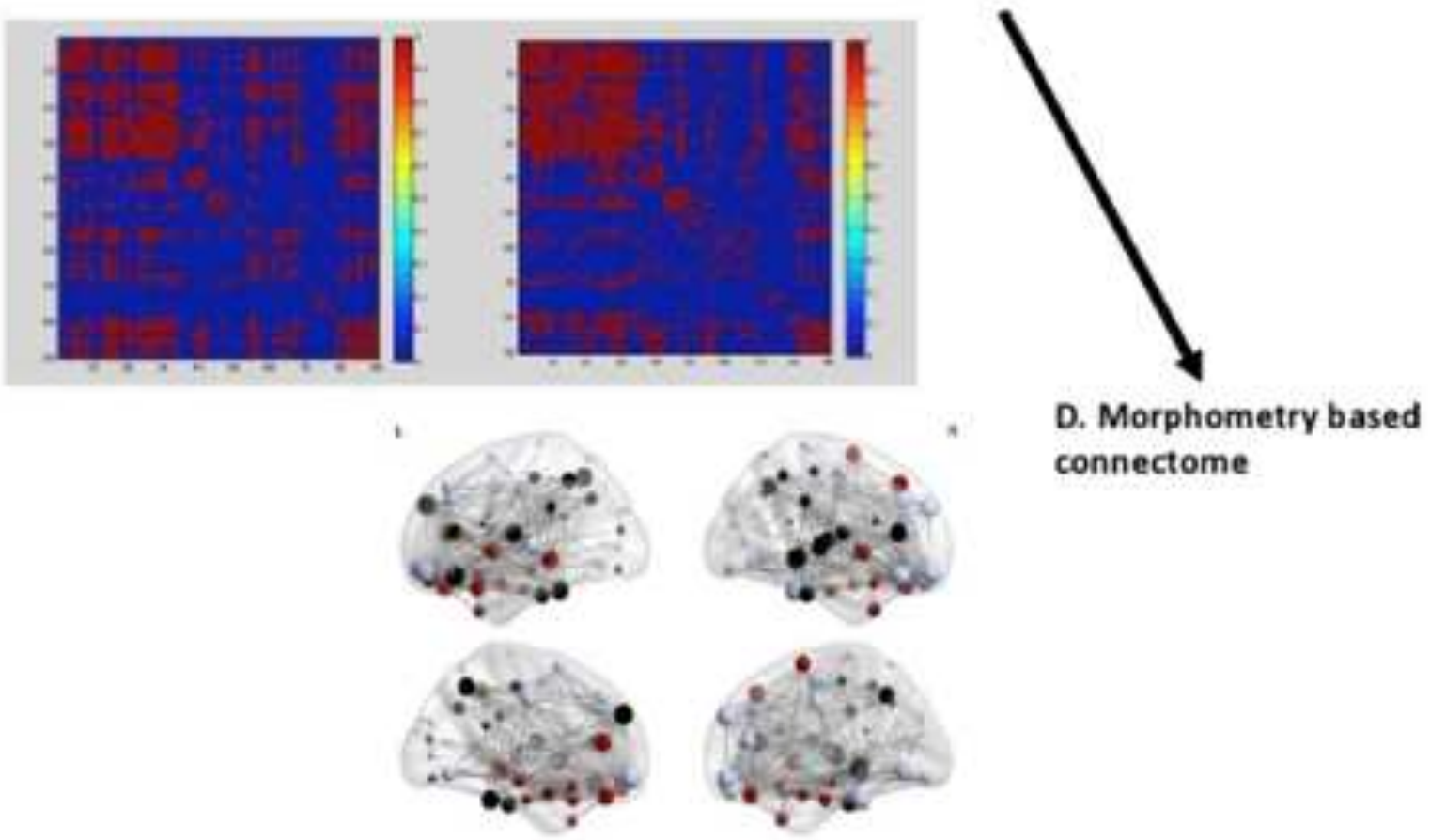
connectome 

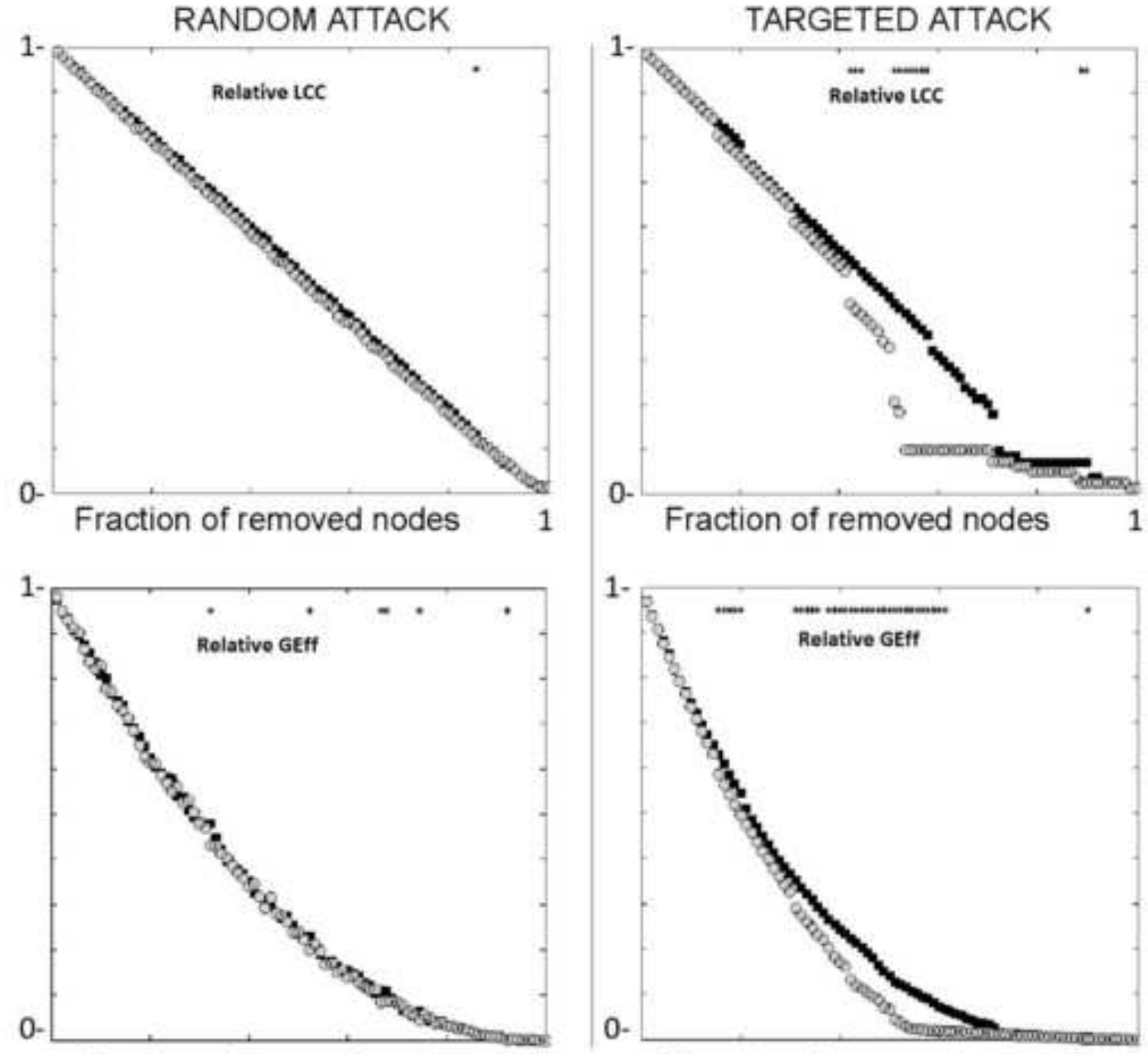

Fraction of removed nodes 1

Fraction of removed nodes

\section{O SCHIZOPHRENIA}
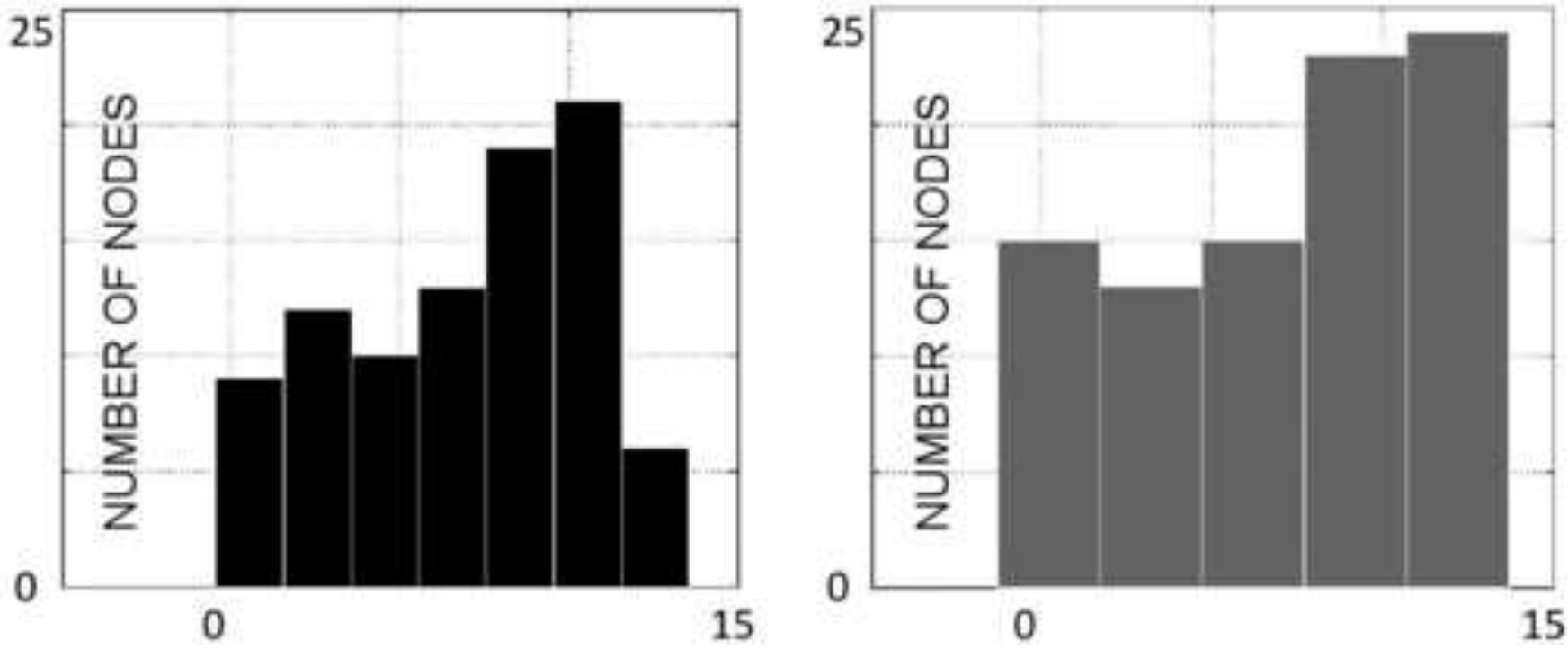
Click here to download Figure(s) Fig3.png $\stackrel{ \pm}{ }$
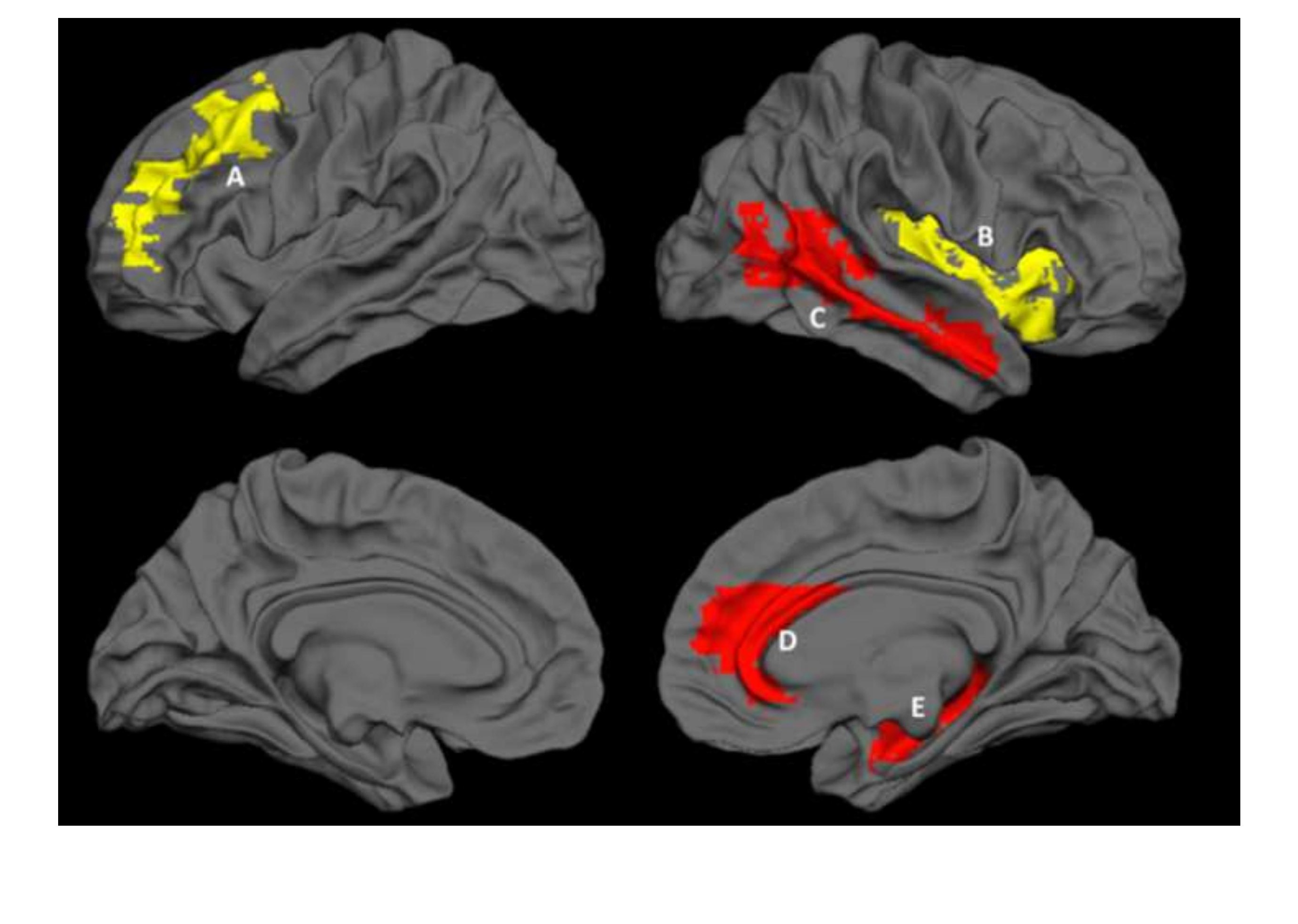
Table 1: Demographic features of the sample

\begin{tabular}{|c|c|c|c|c|}
\hline & $\begin{array}{l}\text { Healthy } \\
\text { controls } \\
(n=40)\end{array}$ & $\begin{array}{l}\text { Patients with } \\
\text { schizophrenia }(n=41)\end{array}$ & $T / X 2$ & $\begin{array}{l}P \\
\text { value }\end{array}$ \\
\hline Age in years (SD) & $33.4(9.1)$ & $33.63(9.2)$ & -0.12 & 0.91 \\
\hline $\begin{array}{l}\text { Gender } \\
\text { (male/female) }\end{array}$ & $29 / 11$ & $31 / 10$ & 0.13 & 0.82 \\
\hline $\begin{array}{l}\text { Handedness } \\
\text { (right/left) }\end{array}$ & $36 / 4$ & $37 / 4$ & 0.001 & 0.97 \\
\hline $\begin{array}{l}\text { Mean parental NS- } \\
\text { SEC (SD) }\end{array}$ & $2.00(1.3)$ & $2.46(1.5)$ & 1.46 & 0.15 \\
\hline SSPI score & - & & & \\
\hline Total & & $11.7(7.4)$ & & \\
\hline Reality Distortion & - & $2.24(2.6)$ & & \\
\hline Disorganisation & - & $1.34(1.3)$ & & \\
\hline $\begin{array}{l}\text { Psychomotor } \\
\text { Poverty }\end{array}$ & - & $2.88(3.8)$ & & \\
\hline
\end{tabular}

NS-SEC: National Statistics-Socio Economic Classification; SSPI, Signs and Symptoms of Psychotic Illness SD: Standard deviation 
Table 2: Topological properties of gray matter-based connectome

\begin{tabular}{|c|c|c|c|}
\hline & Controls & Schizophrenia & $\begin{array}{l}\text { FDA } \\
\text { permutation } p \\
\text { values }\end{array}$ \\
\hline \multicolumn{4}{|l|}{ Measures of segregation } \\
\hline Mean Clustering Coefficient & 0.6799 & 0.7463 & 0.016 \\
\hline \multicolumn{4}{|l|}{ Measures of Integration } \\
\hline Global Efficiency & 0.6705 & 0.6176 & 0.064 \\
\hline \multicolumn{4}{|l|}{ Measures of resilience } \\
\hline \multicolumn{4}{|l|}{ Targeted Attack } \\
\hline $\begin{array}{l}\text { Mean relative size of } \\
\text { remaining large component }\end{array}$ & $43.7 \%$ & $37.5 \%$ & 0.030 \\
\hline $\begin{array}{l}\text { Mean relative global } \\
\text { efficiency }\end{array}$ & $25.7 \%$ & $22.3 \%$ & 0.030 \\
\hline \multicolumn{4}{|l|}{ Random Attack } \\
\hline $\begin{array}{l}\text { Mean relative size of } \\
\text { remaining large component }\end{array}$ & $48.8 \%$ & $47.9 \%$ & 0.262 \\
\hline $\begin{array}{l}\text { Mean relative global } \\
\text { efficiency }\end{array}$ & $32.2 \%$ & $31.8 \%$ & 0.368 \\
\hline
\end{tabular}

FDA - Functional Data Analysis. Reported values are means across all cost densities. FDA comparisons are based on fitting a curve across all cost densities for each group and comparing the shape of the curves. 
Table 3: Hubs in healthy controls and schizophrenia

\begin{tabular}{cc}
\hline Hubs in Controls & Hubs in Schizophrenia \\
\hline $\begin{array}{c}\text { Frontal regions } \\
\text { Frontal Superior Orbital (Left and }\end{array}$ & \\
Frontal Middle Orbital (Right) & Frontal Superior Orbital (Right) \\
Frontal Inferior Orbital (Right) & Frontal Middle Orbital (Left) \\
Frontal Medial Superior (Right) & Frontal Medial Superior (Right and Left) \\
Frontal Medial Orbital (Right) & Frontal Medial Orbital (Right and Left) \\
Frontal Middle (Right) & Frontal Middle (Right) \\
Rectal gyrus (Right and Left) \\
Anterior Cingulate (Left) \\
Rest of the brain \\
Temporal Superior (Left) \\
Temporal Middle (Right) \\
Insula (Left) \\
-
\end{tabular}

Regions in block letters are observed to be hubs in one group only. 
Table S1. Modularity in patients with schizophrenia and healthy controls

\begin{tabular}{|c|c|c|c|c|}
\hline \multicolumn{5}{|c|}{ Modules in healthy controls } \\
\hline Module 1 & Module 2 & Module 3 & Module 4 & Module 5 \\
\hline $\begin{array}{l}\text { Precentral_L } \\
\text { Precentral_R } \\
\text { Frontal_Sup_L } \\
\text { Frontal_Sup_R } \\
\text { Frontal_Sup_Orb_L } \\
\text { Frontal_Sup_Orb_R } \\
\text { Frontal_Mid_L } \\
\text { Frontal_Mid_R } \\
\text { Frontal_Mid_Orb_L } \\
\text { Frontal_Inf_Oper_L } \\
\text { Frontal_Inf_Oper_R } \\
\text { Frontal_Inf_Tri_L } \\
\text { Frontal_Inf_Tri_R } \\
\text { Frontal_Inf_Orb_L } \\
\text { Frontal_Inf_Orb_R } \\
\text { Rolandic_Oper_L } \\
\text { Rolandic_Oper_R } \\
\text { Supp_Motor_Area_L } \\
\text { Supp_Motor_Area_R } \\
\text { Frontal_Sup_Medial_L } \\
\text { Frontal_Sup_Medial_R } \\
\text { Frontal_Med_Orb_L } \\
\text { Insula_L } \\
\text { Insula_R } \\
\text { Cingulum_Ant_L } \\
\text { Cingulum_Ant_R } \\
\text { Cingulum_Mid_L } \\
\text { Cingulum_Mid_R } \\
\text { Lingual_L } \\
\text { Parietal_Sup_R } \\
\text { Paracentral_Lobule_L } \\
\text { Paracentral_Lobule_R } \\
\text { Heschl_L } \\
\text { Heschl_R } \\
\text { Temporal_Sup_L }\end{array}$ & $\begin{array}{l}\text { Olfactory_L } \\
\text { Olfactory_R } \\
\text { Frontal_Med_Orb_R } \\
\text { Rectus_L } \\
\text { Rectus_R } \\
\text { Hippocampus_L } \\
\text { Hippocampus_R } \\
\text { ParaHippocampal_L } \\
\text { ParaHippocampal_R } \\
\text { Amygdala_L } \\
\text { Amygdala_R } \\
\text { Fusiform_L } \\
\text { Fusiform_R } \\
\text { Caudate_L } \\
\text { Caudate_R } \\
\text { Temporal_Pole_Sup_L } \\
\text { Temporal_Pole_Sup_R } \\
\text { Temporal_Mid_L } \\
\text { Temporal_Mid_R } \\
\text { Temporal_Pole_Mid_L } \\
\text { Temporal_Pole_Mid_R } \\
\text { Temporal_Inf_L } \\
\text { Temporal_Inf_R }\end{array}$ & $\begin{array}{l}\text { Calcarine_L } \\
\text { Calcarine_R } \\
\text { Cuneus_L } \\
\text { Cuneus_R } \\
\text { Lingual_R } \\
\text { Occipital_Sup_L } \\
\text { Occipital_Sup_R }\end{array}$ & $\begin{array}{l}\text { Frontal_Mid_Orb_R } \\
\text { Cingulum_Post_L } \\
\text { Cingulum_Post_R } \\
\text { Occipital_Mid_L } \\
\text { Occipital_Mid_R } \\
\text { Occipital_Inf_L } \\
\text { Occipital_Inf_R } \\
\text { Postcentral_L } \\
\text { Postcentral_R } \\
\text { Parietal_Sup_L } \\
\text { Parietal_Inf_L } \\
\text { Parietal_Inf_R } \\
\text { SupraMarginal_L } \\
\text { SupraMarginal_R } \\
\text { Angular_L } \\
\text { Angular_R } \\
\text { Precuneus_L } \\
\text { Precuneus_R } \\
\text { Temporal_Sup_R }\end{array}$ & $\begin{array}{l}\text { Putamen_L } \\
\text { Putamen_R } \\
\text { Pallidum_L } \\
\text { Pallidum_R } \\
\text { Thalamus_L } \\
\text { Thalamus_R }\end{array}$ \\
\hline
\end{tabular}

Palaniyappan et al. 


\begin{tabular}{|c|c|c|c|c|c|c|}
\hline \multicolumn{7}{|c|}{ Modules in patients with schizophrenia } \\
\hline Module 1 & Module 2 & Module 3 & Module 4 & Module 5 & Module 6 & Module 7 \\
\hline $\begin{array}{l}\text { Frontal_Inf_Oper_L } \\
\text { Frontal_Inf_Oper_R } \\
\text { Frontal_Inf_Tri_L } \\
\text { Frontal_Inf_Tri_R } \\
\text { Frontal_Inf_Orb_L } \\
\text { Rolandic_Oper_L } \\
\text { Rolandic_Oper_R } \\
\text { Frontal_Sup_Medial_L } \\
\text { Cingulum_Post_L } \\
\text { Cingulum_Post_R } \\
\text { Occipital_Mid_L } \\
\text { Occipital_Inf_L } \\
\text { Fusiform_L } \\
\text { Postcentral_L } \\
\text { Postcentral_R } \\
\text { Parietal_Inf_L } \\
\text { Parietal_Inf_R } \\
\text { SupraMarginal_L } \\
\text { SupraMarginal_R } \\
\text { Angular_L } \\
\text { Angular_R } \\
\text { Precuneus_L } \\
\text { Precuneus_R } \\
\text { Heschl_L } \\
\text { Heschl_R } \\
\text { Temporal_Sup_R } \\
\text { Temporal_Mid_R } \\
\text { Temporal_Inf_L } \\
\text { Temporal_Inf_R }\end{array}$ & $\begin{array}{l}\text { Precentral_L } \\
\text { Precentral_R } \\
\text { Frontal_Sup_L } \\
\text { Frontal_Sup_Orb_R } \\
\text { Frontal_Mid_L } \\
\text { Frontal_Mid_R } \\
\text { Frontal_Mid_Orb_L } \\
\text { Frontal_Mid_Orb_R } \\
\text { Frontal_Inf_Orb_R } \\
\text { Supp_Motor_Area_L } \\
\text { Frontal_Sup_Medial_R } \\
\text { Frontal_Med_Orb_L } \\
\text { Frontal_Med_Orb_R } \\
\text { Cingulum_Ant_R } \\
\text { Cingulum_Mid_L } \\
\text { Cingulum_Mid_R } \\
\text { Calcarine_L } \\
\text { Calcarine_R } \\
\text { Cuneus_L } \\
\text { Cuneus_R } \\
\text { Lingual_L } \\
\text { Lingual_R } \\
\text { Occipital_Sup_L } \\
\text { Occipital_Sup_R } \\
\text { Occipital_Mid_R } \\
\text { Occipital_Inf_R } \\
\text { Fusiform_R } \\
\text { Paracentral_Lobule_L } \\
\text { Paracentral_Lobule_R } \\
\text { Temporal_Sup_L }\end{array}$ & $\begin{array}{l}\text { Parietal_Sup_L } \\
\text { Parietal_Sup_R }\end{array}$ & $\begin{array}{l}\text { Caudate_L } \\
\text { Caudate_R }\end{array}$ & $\begin{array}{l}\text { Putamen_L } \\
\text { Putamen_R } \\
\text { Pallidum_L } \\
\text { Pallidum_R }\end{array}$ & $\begin{array}{l}\text { Thalamus_L } \\
\text { Thalamus_R }\end{array}$ & $\begin{array}{l}\text { Frontal_Sup_R } \\
\text { Frontal_Sup_Orb_L } \\
\text { Supp_Motor_Area_R } \\
\text { Olfactory_L } \\
\text { Olfactory_R } \\
\text { Rectus_L } \\
\text { Rectus_R } \\
\text { Insula_L } \\
\text { Insula_R } \\
\text { Cingulum_Ant_L } \\
\text { Hippocampus_L } \\
\text { Hippocampus_R } \\
\text { ParaHippocampal_L } \\
\text { ParaHippocampal_R } \\
\text { Amygdala_L } \\
\text { Amygdala_R } \\
\text { Temporal_Pole_Sup_L } \\
\text { Temporal_Pole_Sup_R } \\
\text { Temporal_Mid_L } \\
\text { Temporal_Pole_Mid_L } \\
\text { Temporal_Pole_Mid_R }\end{array}$ \\
\hline
\end{tabular}




\section{Supplemental Section: Effect of antipsychotics on topological properties}

A number of observations from experimental animal studies ${ }^{4}$ and human observational studies from adults with schizophrenia ${ }^{5-8}$ have indicated that certain antipsychotics may contribute to progressive loss of brain tissue. The evidence is less conclusive for atypical antipsychotics 7,9 . In the current sample, all patients received atypical antipsychotics.

Cumulative exposure to antipsychotics is likely to be more influential on the brain morphology than the current stable dose. In the current study, we did not have the longitudinal information on the exact cumulative dose prescription or intake before the scans. Further, we also lacked any data on the individual concordance levels of the prescribed antipsychotics. Therefore, in line with our previous studies ${ }^{11,12}$, we approximated the cumulative antipsychotic exposure, using a product of define daily dose (DDD) and duration of illness since the time of first presentation with psychotic episode, determined from patients' case notes. This index can be taken as an approximate measure of lifetime antipsychotic exposure (ALAE).

We sought to study the relationship between structural covariance and cumulative antipsychotic dose exposure (ALAE) using a liberal threshold of $p=0.1$, with no correction for multiple testing.

To further delineate the effect of approximate lifetime exposure of antipsychotics on topology of covariance, we constructed two association matrices for patient sample thresholded at minimum density for full connectivity. The first association matrix was obtained without adjusting for the effect of approximate lifetime exposure of antipsychotic dose. The second matrix was obtained after linearly adjusting for the effect of approximate lifetime exposure of antipsychotic dose (i.e. regressing out across-subject differences in ALAE in the patient sample and using residuals to compute $90 * 90$ correlation matrix). From these two change matrices, we derived a differential matrix $(\Delta r)$ by subtracting one from the other. Of the 4005 cells $(90 * 89 / 2)$, less than $5 \%$ had a difference in coefficients $>0.1$, suggesting that the effect of lifetime antipsychotic exposure on structural covariance affects a small number of associations. 
A

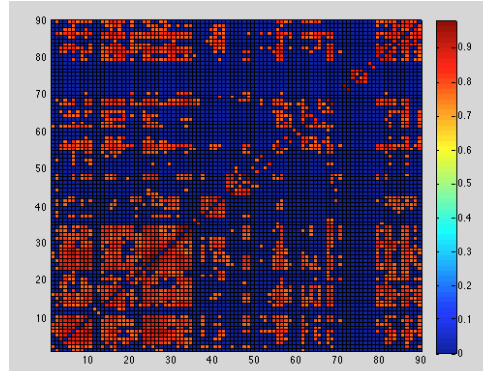

B

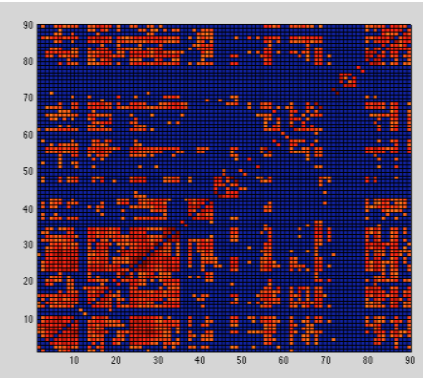

C

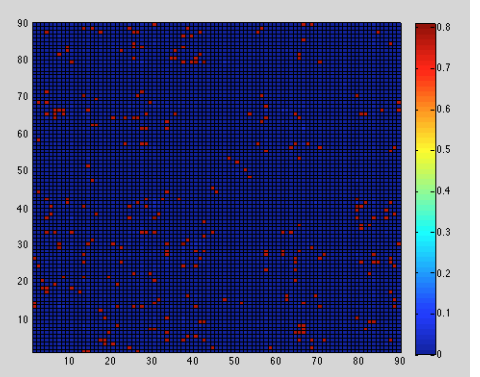

Figure S1: Effect of approximate lifetime exposure of antipsychotics on topology of covariance: Correlation matrices for patient sample thresholded at minimum density for full connectivity. A) Association matrix without adjusting for the effect of approximate lifetime exposure of antipsychotic dose. B) Matrix linearly adjusted for the effect of approximate lifetime exposure of antipsychotic dose. Colour bar indicates absolute correlation coefficients (varying from 0 to 1). C) Absolute matrix obtained from subtracting $A$ and $B(\Delta r)$.

Though a large number of pairwise correlations were relatively unaffected by ALAE, to further clarify if this subtle effect of antipsychotic exposure has indeed any effect on the topological properties of the covariance matrix, we obtained the global network topological measures at the minimum density of full connectivity for both ALAEadjusted and non-adjusted networks. Comparison of these values using the same permutation approach described in the manuscript did not reveal any significant differences between the two networks for small-worldness, mean global and global efficiency, clustering coefficient, and targeted and random attack metrics (all $p>0.39$ ). These comparisons were undertaken without FDR correction to enable detection of even weak effects. 
Table S2: Effect of antipsychotics on topological properties

$\begin{array}{ll}\text { Network unadjusted } & \text { Network } \\ \text { for ALAE } & \text { linearly } \\ & \text { adjusted for } \\ & \text { ALAE }\end{array}$

\begin{tabular}{lcc}
\hline $\begin{array}{l}\text { Measures of Segregation } \\
\text { Clustering Coefficient }\end{array}$ & 0.7463 & 0.7476 \\
\hline $\begin{array}{l}\text { Measures of Integration } \\
\text { Global Efficiency }\end{array}$ & 0.6176 & 0.6207 \\
\hline Measures of resilience & & \\
$\begin{array}{l}\text { Targeted Attack } \\
\text { Mean relative size of remaining } \\
\text { large component }\end{array}$ & $37.5 \%$ & $36.7 \%$ \\
$\begin{array}{l}\text { Mean relative global efficiency } \\
\text { Random Attack }\end{array}$ & $22.3 \%$ & $22.2 \%$ \\
$\begin{array}{l}\text { Mean relative size of remaining } \\
\text { large component }\end{array}$ & $47.9 \%$ & $47.8 \%$ \\
Mean relative global efficiency & $31.8 \%$ & $31.5 \%$ \\
\hline
\end{tabular}

In summary, the negative results despite our extensive approach to relate available antipsychotic treatment data to covariance of longitudinal changes suggests that the reported topology of covariance is unlikely to be due antipsychotic use. Of note, while a number of rigorous studies have examined the effect of antipsychotics on structural changes in schizophrenia, to our knowledge there are no reports on how cumulative antipsychotic exposure affects the structural covariance among various brain regions in schizophrenia. 


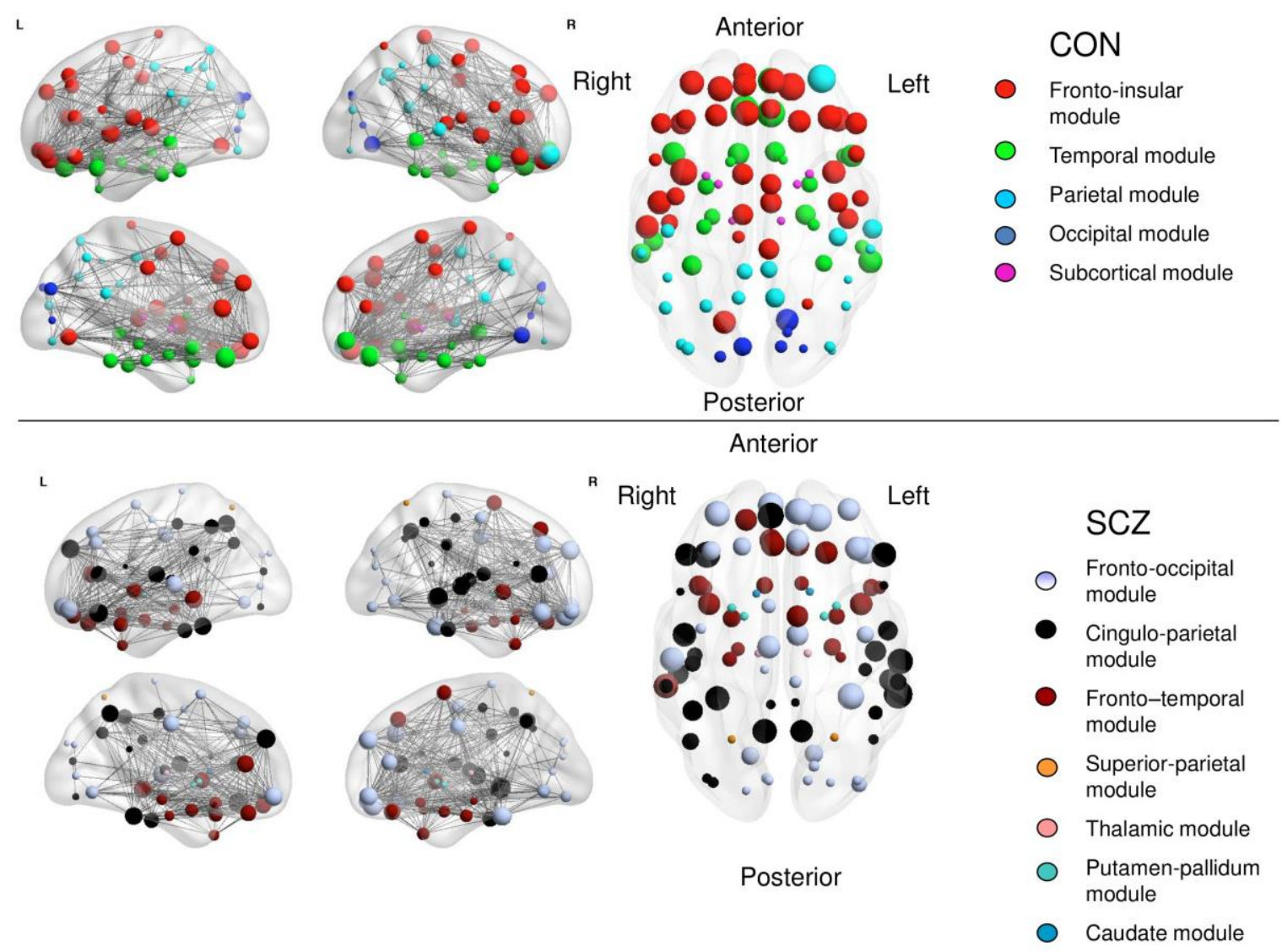

Figure S2: Graphical representation of gray matter connectomes. Connectomes in controls and schizophrenic patients are visualized using BrainNet viewer (www.nitrc.org/projects/bnv). The modules are color-coded separately for each network in the online version of this image. The size of the nodes is proportional to the nodal degree (number of edges) within each connectome. 


\section{Supplemental Section: Voxel Based Morphometric Differences between controls and patients with schizophrenia}

The whole-brain grey matter VBM analyses revealed 13 clusters which were reduced in patients with schizophrenia compared to healthy controls using an uncorrected threshold of $p<.01$ with a cluster extent of 100 voxels. Largest clusters were found in the cingulate gyrus, thalamus, inferior frontal gyrus, insula and hippocampus. Other regions of reduced grey matter were also found in temporal regions, parahippocampal gyrus and also the postcentral gyrus. These results are summarized in Table S3 and Figure S3 below.

Table S3. VBM results for grey matter volume differences between schizophrenia patients and healthy controls $(p<.01$ (uncorrected), $k=100)$. There were no regions with significant tissue increase in patients compared to controls.

\begin{tabular}{|c|c|c|c|c|c|c|}
\hline \multirow[t]{2}{*}{ Peak region } & \multirow[t]{2}{*}{ L/R } & \multicolumn{3}{|c|}{$\begin{array}{l}\text { Peak MNI coordinates } \\
(\mathrm{mm})\end{array}$} & \multirow{2}{*}{$\begin{array}{l}\text { Cluster } \\
\text { extent } \\
\text { (voxels) }\end{array}$} & \multirow[t]{2}{*}{$\begin{array}{l}\text { Peak T } \\
\text { value }\end{array}$} \\
\hline & & $\mathbf{x}$ & $\mathbf{y}$ & $\mathbf{z}$ & & \\
\hline Middle cingulate gyrus & $\mathrm{L}$ & -3 & -33 & 38 & 12284 & 4.51 \\
\hline Thalamus & $\mathrm{L}$ & -2 & -15 & 5 & 648 & 3.5 \\
\hline Inferior frontal gyrus & $\mathrm{L}$ & -41 & 29 & -6 & 896 & 3.44 \\
\hline Insula & $\mathrm{R}$ & 51 & 12 & -2 & 1047 & 3.38 \\
\hline Middle temporal gyrus & $\mathrm{L}$ & -53 & -60 & 14 & 345 & 3.38 \\
\hline Superior frontal gyrus & $\mathrm{R}$ & 17 & 56 & 24 & 504 & 3.32 \\
\hline Postcentral gyrus & $\mathrm{L}$ & -57 & -8 & 33 & 272 & 3.06 \\
\hline Hippocampus & $\mathrm{L}$ & -30 & -15 & -17 & 662 & 3.04 \\
\hline Superior temporal gyrus & $\mathrm{L}$ & -60 & -2 & 8 & 246 & 2.95 \\
\hline Parahippocampal gyrus & $\mathrm{R}$ & 20 & -3 & -29 & 438 & 2.89 \\
\hline Middle temporal gyrus & $\mathrm{L}$ & -50 & -59 & -3 & 236 & 2.89 \\
\hline Insula & $\mathrm{L}$ & -44 & -11 & 5 & 161 & 2.81 \\
\hline Superior temporal gyrus & $\mathrm{R}$ & 56 & -29 & 17 & 162 & 2.75 \\
\hline
\end{tabular}




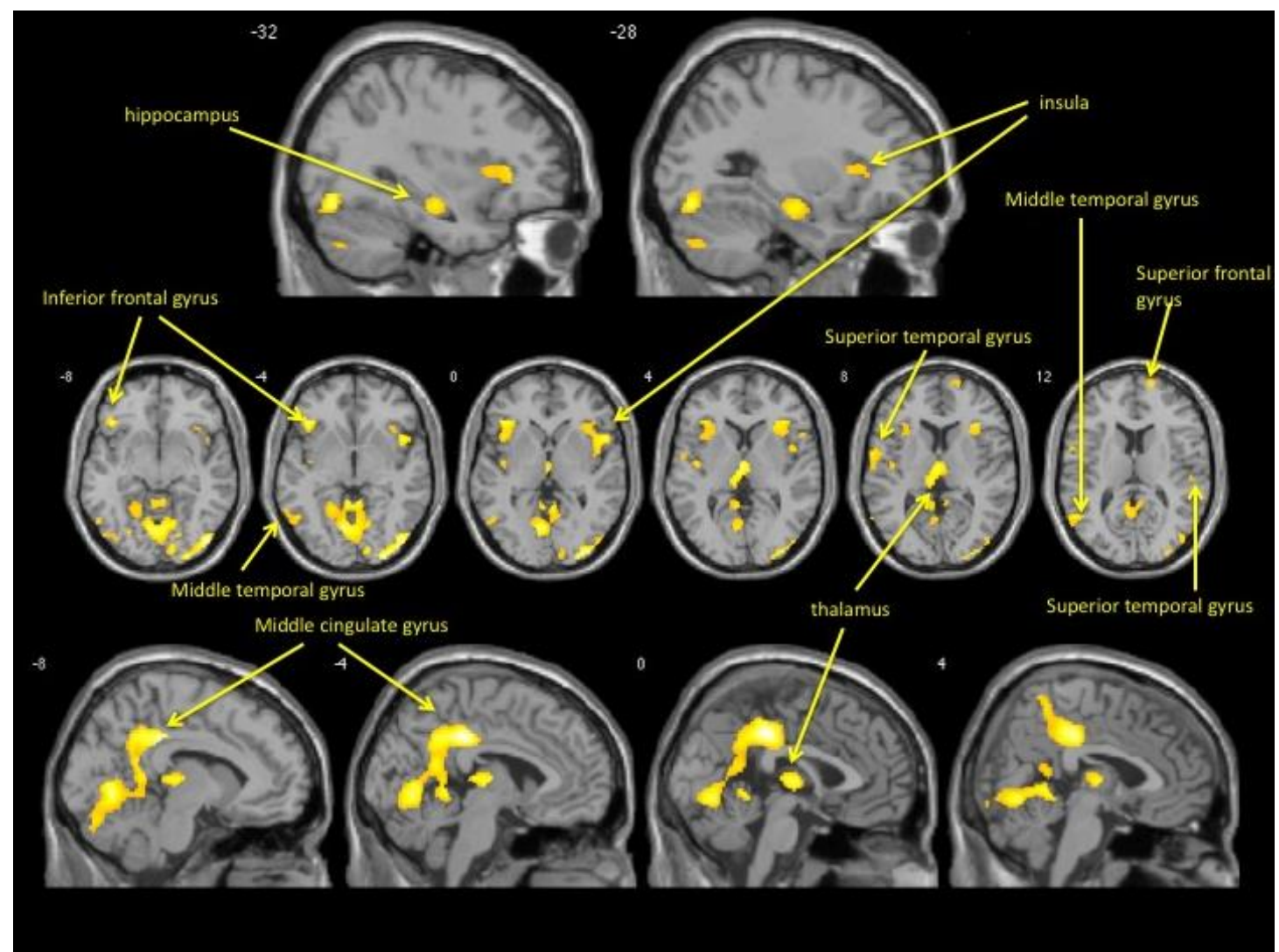

Figure S3. VBM results for grey matter volume differences between healthy controls $>$ schizophrenia patients. For display purposes, regions surviving a threshold of $p<.01$ and cluster extent of $k=100$ are shown on selected slices of a T1 single subject template using MRICron. 


\section{References}

Dorph-Petersen K-A, Pierri JN, Perel JM, Sun Z, Sampson AR, \& Lewis DA (2005). The Influence of Chronic Exposure to Antipsychotic Medications on Brain Size before and after Tissue Fixation: A Comparison of Haloperidol and Olanzapine in Macaque Monkeys. Neuropsychopharmacology 30, 1649-1661.

Fusar-Poli P, Smieskova R, Kempton MJ, Ho BC, Andreasen NC, \& Borgwardt $\mathbf{S}$ (2013). Progressive brain changes in schizophrenia related to antipsychotic treatment? A meta-analysis of longitudinal MRI studies. Neuroscience \& Biobehavioral Reviews 37, 1680-1691.

Lieberman JA, Tollefson GD, Charles C, Zipursky R, Sharma T, Kahn RS, Keefe RSE, Green Al, Gur RE, McEvoy J, Perkins D, Hamer RM, Gu H, Tohen M, \& for the HGDH Study Group (2005). Antipsychotic Drug Effects on Brain Morphology in First-Episode Psychosis. Arch Gen Psychiatry 62, 361-370.

Navari S, \& Dazzan P (2009). Do antipsychotic drugs affect brain structure? A systematic and critical review of MRI findings. Psychological Medicine 39, 17631777.

Palaniyappan L, Park B, Balain V, Dangi R, \& Liddle P (2014). Abnormalities in structural covariance of cortical gyrification in schizophrenia. Brain structure \& function

Roiz-Santiañez R, Suarez-Pinilla P, \& Crespo-Facorro B (2015). Brain Structural Effects of Antipsychotic Treatment in Schizophrenia: A Systematic Review. Current Neuropharmacology 13, 422-434.

Smieskova R, Fusar-Poli P, Allen P, Bendfeldt K, Stieglitz RD, Drewe J, Radue EW, McGuire PK, Riecher-Rössler A, \& Borgwardt SJ (2009). The effects of antipsychotics on the brain: what have we learnt from structural imaging of schizophrenia?--a systematic review. Current Pharmaceutical Design 15, 25352549.

Torres US, Duran FLS, Schaufelberger MS, Crippa JAS, Louzã MR, Sallet PC, Kanegusuku CYO, Elkis H, Gattaz WF, Bassitt DP, Zuardi AW, Hallak JEC, Leite CC, Castro CC, Santos AC, Murray RM, \& Busatto GF (2016). Patterns of regional gray matter loss at different stages of schizophrenia: A multisite, cross-sectional VBM study in first-episode and chronic illness. Neurolmage : Clinical 12, 1-15. 
Lena Palaniyappan, MD, PhD, FRCPC

Department of Psychiatry

University of Western Ontario

London, ON. Canada

Email: Ipalaniy@uwo.ca

$24^{\text {th }}$ January 2018

Dear Prof. Murray \& the editorial team,

Please find the revised version of the manuscript 'Structural covariance and cortical reorganization in schizophrenia: A MRI-based morphometric study'.

We are grateful for providing us an opportunity to revise this work. We have addressed all of the points raised by the reviewers, and attach a document outlining the changes made. We hope that you will find the manuscript acceptable for publication.

Thank you very much for your consideration. I look forward to hear from you.

Yours sincerely,

Dr. Lena Palaniyappan, MD, PhD, FRCPC.

Corresponding author 